Siberian Branch of Russian Academy of Science Budker Institute of Nuclear Physics

\author{
V. A. Chernov, N. V. Kovalenko, \\ S. V. Mytnichenko, A. I. Toropov \\ FINE STRUCTURE \\ OF X-RAY DIFFUSE SCATTERING \\ IN THE VICINITY OF HIGH-ANGLE \\ SUPERLATTICE BRAGG REFLECTIONS
}

Budker INP 2003-12

Novosibirsk

2003 


\title{
Fine structure of X-ray diffuse scattering in the vicinity of high-angle superlattice Bragg reflections
}

\author{
V. A. Chernov \\ Boreskov Institute of Catalysis, Novosibirsk, Russia \\ Siberian SR Center at Budker Institute of Nuclear Physics \\ 630090 Novosibirsk, Russia \\ N. V. Kovalenko \\ Budker Institute of Nuclear Physics \\ 630090 Novosibirsk, Russia \\ S. V. Mytnichenko \\ Institute of Solid State Chemistry \\ Siberian SR Center at Budker Institute of Nuclear Physics \\ 630090 Novosibirsk, Russia \\ A. I. Toropov \\ Institute of Semiconductor Physics \\ 630090 Novosibirsk, Russia
}

\begin{abstract}
Triple axis X-ray diffractometry was used to study diffuse scattering from an AlAs/GaAs superlattice grown on an [001]-oriented GaAs substrate by molecular beam epitaxy. Diffraction space maps were obtained around (002) reflection and its low-angle first-order satellite. The data obtained reveal not only usual quasi-Bragg diffuse scattering sheets, but also amplification of diffuse scattering when the incoming or outgoing angle is nearly equal to the superlattice or substrate Bragg angle. The observed domination degree in the intensity of the incoming features over the outgoing ones was shown to reflect the decay rate of coherent X-ray field through the diffuse scattering channel.
\end{abstract}


X-ray diffuse scattering from multilayers and superlattices was the object of study of a large body of work in the last decade. Significant scientific progress in X-ray diffuse scattering from amorphous multilayers was made during this time. It starts with Refs. 1-5 in which the coherent replication of rough multilayer interfaces was shown to cause resonant amplification of diffuse scattering resulting in the observation of "quasi-Bragg" diffuse scattering. One more of the diffraction effects is resonant amplification of diffuse scattering when the incoming or outgoing angle is nearly equal to the Bragg angle. For the first time this effect was observed experimentally by Kortright and co-worker [3, 6] and by Savage et al [4]. It was qualitatively explained as standing-wave effect for the incident and diffusely scattered X-ray fields. Numerical theoretical calculations of phenomenon under discussion were performed by extending the distorted wave Born approximation (DWBA), previously used to calculate diffuse scattering from single surfaces [7], to the case of multilayers [8].

A case of X-ray diffuse scattering from superlattices is more complicated. One of the most important reasons of this is an inevitable presence of terrace structure [9-15]. As a result both the interfacial roughness and the lattice strain become anisotropic in the lateral directions resulting in the observation of the diffuse scattering dependence on the azimuthal sample orientation. Note that in contrast to the case of small-angle Bragg reflections from multilayers, where diffuse scattering is only caused by electron density fluctuations in lateral directions (interfacial roughness), in the case of the lattice Bragg reflections from superlattices and their satellites diffuse scattering is also caused by lateral inhomogeneity of lattice strain. Furthermore, in contrast to the case of nonepitaxial multilayers, replication manner of interfacial profiles trough the layer stack can be more intricate in superlattices $[11,12,15]$. Nevertheless, the 
interest in this method was recently increased, especially in connection with a creation of novel semiconductor devices such as quantum dots or wires [16-19].

In this article we report an observation of features of X-ray diffuse scattering from an $\mathrm{AlAs} / \mathrm{GaAs}$ superlattice when the incoming or outgoing angle is nearly equal (within Darwin's table) to the high-angle superlattice or substrate Bragg angle. The similar effect was previously observed for amorphous multilayers $[3,4,6,20]$.

A $\left[(\mathrm{AlAs})_{9 \mathrm{ML}} /(\mathrm{GaAs})_{9 \mathrm{ML}}\right] \times 80$ superlattice was grown by molecular beam epitaxy on [001]-oriented GaAs substrate with a buffer AlAs layer $(\sim 50 \mathrm{~nm})$ in Riber 32P system. The growth was monitored by reflection high-energy electron diffraction. X-ray diffraction measurements were performed using synchrotron radiation (SR) from the VEPP-3 storage ring and using a triple axis diffractometer with a primary channel-cut single-crystal $\mathrm{Si}(111)$ monochromator and a $\mathrm{Ge}(111)$ crystal-collimator at the wavelength $\lambda=0.145 \mathrm{~nm}$. The measured value of the miscut angle was about $0.23^{\circ}$ and the miscut direction was slightly $\left(\sim 5^{\circ}\right)$ off from the [110] direction. The measurements were performed in such manner that the specular diffraction plane defined by the incident and reflected wave vectors was along the [110] crystallographic direction across terrace steps. A specular scan through the (002) lattice reflection is shown in Fig. 1.

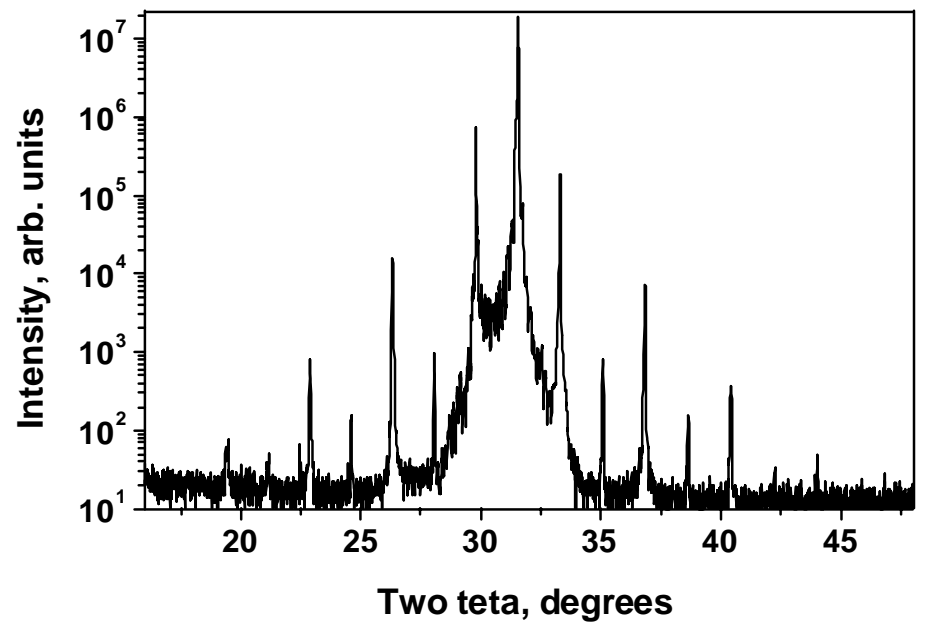

Fig. 1. Specular scan through the (002) reflection. 

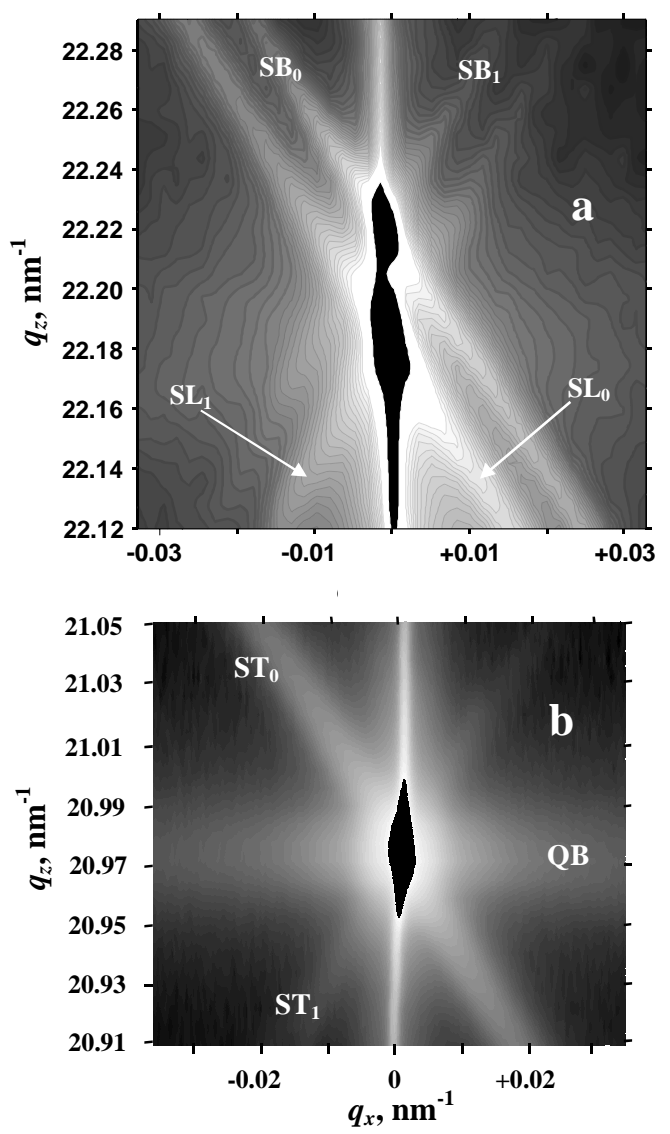

Fig. 2. Diffraction maps of the (002) reflection (a) and its low-angle first-order satellite (b). The in-plane projection of the momentum transfer plotted parallel to the horizontal axis. The momentum transfer normal to lateral planes is plotted parallel to the vertical axis. The intensity is shown in logarithmic scale. Though the dynamical range of the measurements was about $10^{6}$, in order to clearly show the diffuse scattering fine structure, the high intensity point were cut resulting that the final dynamical range of map was about $10^{4}$. The reported diffuse scattering features are presented as the inclined streaks. The features $\mathrm{SB}_{0,1}$ and $\mathrm{SL}_{0,1}$ correspond to the substrate and basic superlattice Bragg peak, respectively. The features $\mathrm{ST}_{0,1}$ correspond to the satellite Bragg peak. Their indices correspond to two conditions when incoming or outgoing angle is equal to Bragg angle, respectively. The feature marked as QB is well-known quasi-Bragg diffuse scattering 5 . 
(a)
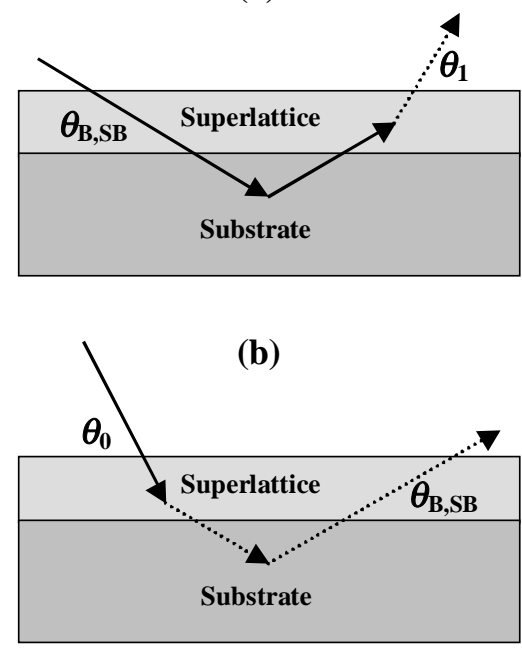

Fig. 3. Origin of $\mathrm{SB}_{0}$ (a) and $\mathrm{SB}_{1}$ (b) features.

The diffraction map of the (002) reflection is shown in Fig. 2(a). The reported diffuse scattering features are marked as $\mathrm{SL}_{0}$ and $\mathrm{SL}_{1}$ corresponding to the superlattice Bragg angle and as $\mathrm{SB}_{0}$ and $\mathrm{SB}_{1}$ corresponding to the substrate Bragg angle. An origin of these features can be more easily explained in the case of substrate Bragg reflection. Two different orders of scattering events are shown in Fig. 3. In the first case the primary beam with the substrate Bragg angle of incidence is reflected by the substrate and then the reflected beam is diffusely scattered by the superlattice generating $\mathrm{SB}_{0}$ (Fig. 3a). However, the order of the events can be inverted. In spite of the angle of incidence of the primary beam, the small-angle diffuse scattering is effectively reflected if its angle of incidence is nearly equal to the substrate Bragg angle generating $\mathrm{SB}_{1}$ (Fig. 3b). These processes have a dynamical nature, at least, because the $\mathrm{SB}_{0}$ and $\mathrm{SB}_{1}$ features can be visible if the substrate Bragg reflectivity is not vanishingly small. Moreover, the standing-wave and other dynamical effects can play an important role in diffraction due to the fact that the sources of diffuse scattering in the superlattice are coherently located relative to the substrate lattice. A similar explanation can be found for the superlattice features $\left(\mathrm{SL}_{0}\right.$ and $\left.\mathrm{SL}_{1}\right)$. In this case the scattering potential can be split into two unequal parts. The first of them is basic and laterally symmetrical. It is respondent for the dynamical specular reflection of 
X-rays. The second is the small disturbance that causes X-ray diffuse scattering. These parts may be considered to play a similar role as the "substrate" and the "superlattice" in the previous case. Though it is impossible to separate the time sequence of the scattering events so clearly as in the case of the substrate Bragg peak, the basic physical mechanism of this phenomenon is the same.

At first sight, the proposed explanation seems to predict that the intensities of the incoming and outgoing features must be equal, respectively. Such symmetry of the diffuse scattering cross section to exchanging the incoming and outgoing angles is known as the reciprocity theorem, which is valid for the case of a conservative system and is a sequence of symmetry of Maxwell's equations relative to time reverse. At the same time, the data obtained (Fig. 2) reveal the superiority in the intensity of the incoming features over the outgoing ones. This discrepancy needs to be discussed.

At first, it is necessary to mark that the reciprocity theorem is not a universal law, which is valid for the description of diffraction from any objects, in particular, of diffraction from multilayers or superlattices. Indeed, interfacial roughness inevitably has the statistical nature, but any statistical system is time irreversible. Therefore, in general the reciprocity theorem can not be applied in this case. This fact can be more clearly explained in terms of the multi-channel scattering theory. The incoherent diffuse scattering can be considered as an additional reaction channel. At the same time, the coherent diffraction can be described by the usual single-channel scattering theory introducing the imaginary corrections to scattering potential that allows one to take into account the dissipation of the coherent filed energy through the incoherent diffuse scattering channel. These corrections automatically cause breakdown of the reciprocity theorem. The corrections are higher the reciprocity theorem breakdown is more evident. Only the Born approximation (including DWBA), where the coherent wave energy is conserved in a forced manner, provides the symmetry under discussion [7]. In contrast to usual photoabsorption, these corrections directly depend on wave propagation direction. Already DWBA predicts that the peak diffuse scattering cross section is achieved at the Bragg angles of propagation. It allows one to explain, why the incoming features dominate over the outgoing features and not the reverse.

The diffraction map of the first-order low-angle satellite of the (002) reflection (Fig. 2b) confirms the conclusion that the asymmetry of the intensity of incoming and outgoing features is caused by the decay of coherent X-ray field 
through the diffuse scattering channel. Indeed, it is possible to show that the energy dissipation is lower in the case of the (002) reflection as compared to the case of its satellite. Though the superlattice interfacial roughness caused by the terraces is the main source of diffuse scattering in all cases, but the mechanism of this phenomenon is different for the case of basic lattice reflections and for the case of satellites. The diffuse scattering around the basic lattice reflection is caused by the lattice strain mainly. But the epitaxial lattice mismatch is small in our case and, furthermore, the lattice strain is effectively dumped by the presence of dislocations. Their influence appears as the wide diffuse halo around the basic lattice reflection in Fig. 1. Thus, the diffuse scattering provided by the lattice strain is relatively weak. The different situation is realized in the case of satellites. The electron density variations at the interfaces influence on diffraction directly. Due to the fact that these variations are well correlated through the layer stack as well as in lateral directions, their contribution to diffuse scattering is higher as compared to the lattice strain. The last conclusion is supported by the evident presence of quasi-Bragg diffuse scattering (QB) in Fig. 2b, whereas this feature is very weak in Fig. 2a. As a result the higher diffuse scattering cross section in the case of the satellite provides that the intensity asymmetry under discussion is more evident in Fig. 2b than in Fig. 2a.

Mark that the similar features around the small-angle Bragg reflections from superlattices [13], around the high-angle Bragg reflections [16] and around the substrate lattice reflections [14, 16-18] were repeatedly observed in literature. Some times these features were explained as an instrumental artifact. Nevertheless, we are sure that our reported features are not an experimental artifact. Firstly, the measurements were accurately performed using the calibrated copper foils in order to measure the intensity in an appropriate tolerance limit of the detector dynamical range. Secondly, the mapping of the (002) reflection from the origin substrate reveals not the features that discussed.

As a rule, in contrast to our data the features observed in the cited works appeared in diffraction maps as the single streaks. By this connection it is necessary to mark the importance of a spatial coherence of incident X-ray beam. For the first time this problem was rose by Sinha et al [21]. In our previous work [20] the high spatial coherence of modern SR sources was shown experimentally to involve the long-range interfacial defects into diffraction. In turn, the longrange defects increase the diffuse scattering cross section drastically [22]. Thus, it is reasonable to expect that the data obtained using SR can reveal the strong 
domination of the incoming diffuse scattering features. The (002) reflection studied in this work has a low structure factor, sequentially, the diffuse scattering cross section is low too. There is one of reasons that allows us to observe the diffuse scattering fine structure as a cross hairs of incoming and outgoing streaks.

In conclusion, we have observed experimentally the resonant features of $\mathrm{X}$-ray diffuse scattering from the $\mathrm{AlAs} / \mathrm{GaAs}$ superlattice when the incoming or outgoing angle is nearly equal to the high-angle superlattice or substrate Bragg angle. The degree of the intensity domination of the incoming feature over the outgoing one was shown to indicate the decay rate of the coherent X-ray field through the diffuse scattering channel.

The present work is submitted to Appl. Phys. Lett.

\section{References}

[1] A.V. Andreev, A. G. Michette, and A. Renwick. J. Mod. Opt. 35, 1667 (1988).

[2] A. Bruson, C. Dufour, B. George, M. Vergant, G. Marchai, and Ph.Mangin. Solid State Commun. 71, 1045 (1989).

[3] J. B. Kortright. J. Appl. Phys. 70, 3620 (1991).

[4] D. E. Savage, J. Kleiner, N. Schimke, Y.-H. Phang, T. Jankowski, J. Jacobs, R. Kariots, and M.G. Lagally. J. Appl. Phys. 69, 1411 (1991).

[5] D. G. Stearns. J. Appl. Phys. 71, 4286 (1992).

[6] J. B. Kortright, and A. Fischer-Colbrie. J. Appl. Phys. 61, 1130 (1987).

[7] S. K. Sinha, E. B. Sirota, S. Garoff, and H. B. Stanley. Phys. Rev. B 38, 2297 (1988).

[8] V. Holý, and T. Baumbach, Phys. Rev. B 49, 10668 (1994).

[9] A. Gibaud, R. A. Cowley, D. F. McMorrow, R. C. C. Ward, and M. R. Wells. Phys. Rev. B 48, 14463 (1993).

[10] S. K. Sinha, M. K. Sanyal, S. K. Satija, C. F. Majkrzak, D. A. Neumann, H. Homma, S. Szpala, A. Gibaud, and H. Morkoc. Physica B 198, 72 (1994).

[11] R. L. Headrick, J.-M. Baribeau, and Y. E. Strausser. Appl. Phys. Lett. 66, 96 (1995). 
[12] B. Jenichen, S. A. Stepanov, B. Brar, and H. Kroemer. J. Appl. Phys. 79, 120 (1996).

[13] E. A. Kondrashkina, S. A. Stepanov, R. Opitz, M. Schimidbauer, R. Kohler, R. Hey, M. Wassermeier, and D. V. Novikov. Phys. Rev. B 56, 10469 (1997).

[14] A. A. Darhuber, J. Zhu, V. Holý, J. Stangl, P. Mikulík, K. Brunner, G. Abstreiter, and G. Bauer. Appl. Phys. Lett. 73, 1535 (1998).

[15] V. Holý, A. A. Darhuber, J. Stangl, G. Bauer, J. Nützel, and G. Abstreiter, Phys. Rev. B57, 12435 (1998).

[16] A. A. Darhuber, E. Koppensteiner, G. Bauer, P. D. Wang, Y. P. Song, C. M. Sotomayor Torres, and M. C. Holland. Appl. Phys. Lett. 66, 947 (1995).

[17] A. A. Darhuber, V. Holy, J. Stangl, G. Bauer, A. Krost, F. Heinrichsdorff, M. Grundman, D. Bimberg, V. M. Ustinov, P. S. Kop'ev, A. O. Kosogov, and P. Werner. Appl. Phys. Lett. 70, 955 (1997).

[18] V. Holý, A. A. Darhuber, J. Stangl, S. Zerlauth, F. Schäffler, G. Bauer, N. Darowski, D. Lübbert, U. Pietsch, and I. Vávra. Phys. Rev. B58, 7934 (1998).

[19] V. Holý, J. Stangl, G. Springholz, M. Pinczolits, G. Bauer, I. Kegel, and T. H. Metzger. Physica B 283, 65 (2000).

[20] V. A. Chernov, V. I. Kondratiev, N. V. Kovalenko, S. V. Mytnichenko, and K. V. Zolotarev. J. Appl. Phys. 92, 7593 (2002).

[21] S. K. Sinha, M. Tolan, and A. Gibaud. Phys. Rev. B57, 2740 (1998).

[22] D. K. G. de Boer, Phys. Rev. B53, 6048 (1996). 


\author{
V. A. Chernov, N. V. Kovalenko,
}

S. V. Mytnichenko, A. I. Toropov

Fine structure of X-ray diffuse scattering in the vicinity of high-angle superlattice Bragg reflections

В. А. Чернов, Н. В. Коваленко,

С. В. Мытниченко, А. И. Торопов

\title{
Тонкая структура рентгеновского диффузного рассеяния вблизи Брэгговских отражений в больших углах от сверхрешеток
}

Budker INP 2003-12

Ответственный за выпуск А.М. Кудрявцев

Работа поступила 7.02. 2003 г.

Сдано в набор 12.02. 2003 г.

Подписано в печать 14.02.2003 г.

Формат 60x90 1/16 Объем 0.8 печ.л., 0.7 уч.-изд.л.

Тираж 85 экз. Бесплатно. Заказ № 12

Обработано на IBM РC и отпечатано

на ротапринте ИЯФ им. Г.И. Будкера СО РАН,

Новосибирск., 630090, пр. Академика Лаврентьева, 11 